\title{
Modalités d'analgésie-sédation procédurale utilisées en traumatologie extrahospitalière par les médecins urgentistes de France métropolitaine
}

\author{
Procedural Sedation Analgesia Modalities Used by Emergency Physicians \\ in French Out-of-Hospital Emergency Medical System
}

\author{
M. Serres · C. Gil-Jardiné · G. Evrard · P. Revel · E. Tentillier · M. Galinski \\ Reçu le 30 mars 2018; accepté le 5 juillet 2018 \\ (C) SFMU et Lavoisier SAS 2018
}

Résumé Introduction : L'analgésie-sédation procédurale (ASP) consiste à administrer des agents sédatifs permettant aux patients de tolérer des procédures désagréables. L'ASP a fait l'objet de recommandations formalisées d'experts de la Société française de médecine d'urgence (SFMU) depuis 2010. L'objectif était d'évaluer l'impact de ces recommandations sur les pratiques des smuristes en France concernant la réalisation d'une ASP.

Méthodes : Enquête téléphonique réalisée de janvier à juillet 2016. Nous avons joint un médecin de permanence le jour de l'appel dans chaque service mobile d'urgence et de réanimation (Smur) métropolitain. Il devait répondre à un questionnaire focalisé sur une situation clinique pour laquelle une ASP était indiquée : fracture fémorale déplacée.

Résultats : Chaque Smur métropolitain a été joint $(n=384)$, et 309 médecins ont répondu au questionnaire (80\%). Quarante-deux combinaisons thérapeutiques différentes ont été proposées, et 308 médecins (99\%) administraient soit un sédatif, soit un opioïde, soit les deux. La morphine était le principal antalgique utilisé $(n=232 ; 75 \%)$, les posologies et les modalités correspondant aux dernières recommandations. La kétamine était le sédatif le plus utilisé $(n=163$; $53 \%$ ), mais les posologies étaient inférieures à celles recommandées. La douleur des patients communicants était éva-

\footnotetext{
M. Serres $(\square)$

Service urgences adultes, centre hospitalier de Dax,

F-40100 Dax, France

e-mail : marine_serres@hotmail.fr

C. Gil-Jardiné · G. Evrard · P. Revel · E. Tentillier · M. Galinski

Pôle urgences adultes, Samu-hôpital Pellegrin,

CHU de Bordeaux, F-33000 Bordeaux, France

C. Gil-Jardiné · P. Revel · M. Galinski

Inserm U1219, Injury Epidemiology Transport Occupation Team, université de Bordeaux-II, F-33076 Bordeaux cedex, France
}

luée avec une échelle adaptée. La douleur des patients non communicants, adultes ou enfants, n'était pas évaluée avec une échelle adaptée. Un tiers des Smur avait un protocole d'ASP, et $27 \%$ des médecins disaient connaître les recommandations. Au total, il y avait une grande hétérogénéité des modalités de réalisation de l'ASP, avec notamment des difficultés de maniement des sédatifs, des recommandations peu connues et un faible taux de protocoles dans les Smur.

Mots clés Soins urgences extrahospitalières - Attitude du personnel soignant $\cdot$ Antalgiques $\cdot$ Hypnotiques et sédatifs

Abstract Introduction: Procedural sedation analgesia (PSA) consists to administrate a sedative drug allowing the patients to support an unpleasant procedure. PSA belongs to French guidelines published by the French Society of Emergency Medicine (SFMU) in 2010. The objective was to assess the impact of SFMU guidelines on the practice of emergency physicians in the emergency and intensive care mobile service (Smur) concerning the realization of PSA.

Methods: Phone survey was realized from January to July 2016. One physician on duty in each Smur was called. He had to answer a questionnaire focalized on a clinical situation for which a PSA was indicated: displaced femoral fracture.

Results: Each metropolitan Smur was called $(N=384)$ and 309 physicians answered the questionnaire (80\%). Forty-two different therapeutic combinations were proposed and 308 $(99 \%)$ physicians administrated either a sedative drug or an opioid or both. Morphine was the main painkiller $(N=232$, $75 \%$ ), doses and modalities corresponding to the recommendations. Ketamine was the most sedative drug administrated $(N=163,53 \%)$ but doses were lower than those of the recommendations. Pain of communicating patients was assessed with a well-adapted scale but it was not the case for non-communicating patients, adults or children. One third of Smurs had a PSA protocol and $27 \%$ of physicians knew the 
recommendations. There was a great heterogeneity of the modalities concerning the realization of PSA, particularly with difficulties to use sedative drugs, a weak knowledge of recommendations and a weak proportion of protocols in the Smurs.

Keywords Prehospital emergency care $\cdot$ Attitude of health personnel $\cdot$ Analgesics $\cdot$ Hypnotics and sedatives

\section{Introduction}

L'analgésie-sédation procédurale (ASP) consiste à administrer des agents sédatifs ou dissociatifs permettant aux patients de tolérer des procédures désagréables. Les gestes les plus communément réalisés en situation d'urgences sont les réductions ou les réalignements de fractures de membre, les réductions de luxations, les cardioversions par choc électrique externe, les drainages d'abcès, tout en conservant une fonction cardiorespiratoire correcte [1]. L'ASP a fait l'objet de recommandations internationales et de recommandations formalisées d'experts (RFE) de la Société française de médecine d'urgence (SFMU) depuis 2010 [1,2]. Les experts avaient recommandé d'anticiper l'analgésie des gestes douloureux chez les patients vigiles par l'administration de morphine par voie intraveineuse, titrée, associée à du mélange équimolaire d'oxygène-protoxyde d'azote (MEOPA) et/ou de la kétamine $(0,5$ à $1 \mathrm{mg} / \mathrm{kg}$ i.v. en titration) [2]. Il était possible d'adjoindre du midazolam à la morphine. Si une sédation profonde était nécessaire, l'utilisation du propofol à faible posologie était recommandée [2]. Les techniques d'anesthésie locale ou d'anesthésie locorégionale (ALR) devaient être utilisées dans la mesure du possible et dans le respect de la conférence d'experts de 2003 [3]. Ils insistaient aussi sur l'importance d'avoir un protocole d'ASP. Une étude récente, multicentrique, réalisée en extrahospitalier montrait que $54 \%$ des patients traumatisés, pris en charge par un Smur, avaient une analgésie-sédation adéquate lors de la réalisation d'un geste douloureux [4]. Elle montrait aussi que les pratiques étaient très hétérogènes et qu'aucun des dix centres participant à l'étude n'avait de protocole.

L'objectif principal de ce travail était d'évaluer l'impact des recommandations françaises concernant l'ASP sur les pratiques des urgentistes travaillant dans des Smur.

\section{Méthode}

Il s'agissait d'une enquête téléphonique réalisée par un seul chercheur, de janvier à juillet 2016. Le médecin du service mobile d'urgence et de réanimation (Smur) de permanence le jour de l'appel dans tous les Smur métropolitains référen- cés dans le Guide des Samu/Smur de France $(n=384)$ était interrogé. Le questionnaire comportait trois parties :

- la première partie portait sur des questions d'ordre général, sur l'interlocuteur (âge, formation et diplômes universitaires supplémentaires) ;

- la deuxième partie portait sur une mise en situation, avec la prise en charge antalgique pratique d'un patient ayant une fracture fémorale en extrahospitalier (techniques d'analgésie-sédation, médicaments, doses) et sur des questions concernant le mode d'évaluation de la douleur et de la sédation, les médicaments disponibles dans l'UMH, la pratique de l'ALR en général, la présence de MEOPA dans les UMH et la connaissance des dernières recommandations ;

- la troisième partie portait sur la présence ou non d'un protocole d'ASP pour le préhospitalier. Les réponses attendues étaient celles présentées par les RFE de la SFMU de 2010 [2].

\section{Analyse statistique}

Les résultats étaient exprimés en moyenne et écart-type pour les données quantitatives et en pourcentage et valeur absolue pour les données qualitatives. Pour le recueil des données et l'analyse, nous avons utilisé le logiciel Excel $^{\circledR} 2010$ version 14.7.4 (Microsoft ${ }^{\circledR}$, Santa Rosa, États-Unis).

\section{Résultats}

Tous les Smur métropolitains ont été joints $(n=384)$, 309 médecins ont pu répondre au questionnaire, soit un taux d'exhaustivité de $80 \%$. Parmi les médecins, 194 (63\%) étaient détenteurs de la capacité de médecine d'urgence, 103 (33\%) possédaient le diplôme d'études spécialisées complémentaires de médecine d'urgence, et 12 (3,9\%) médecins (neuf généralistes, un interniste, un chirurgien maxillofacial et un anesthésiste) n'avaient pas de diplôme spécifique de médecine d'urgence. L'âge moyen des médecins était de 42 ans $( \pm 9)$, avec des extrêmes de 27 et 66 ans.

Pour la prise en charge du patient traumatisé, 98 médecins (32\%) utilisaient du paracétamol, $9(3 \%)$, du kétoprofène et 3 (1\%), du néfopam. La morphine était l'opiö̈de le plus utilisé et la kétamine le sédatif le plus utilisé (Tableau 1). L'analyse des différentes associations thérapeutiques possibles montrait que 42 combinaisons différentes avaient été proposées. À l'exclusion d'un médecin, tous $(n=308$ [99\%]) avaient utilisé soit un sédatif, soit un opioïde ou les deux. Cent quarante-cinq médecins (47 \%) avaient proposé un sédatif et un opioïde associés ou non à un antalgique de palier 1, à du MEOPA ou à une ALR. Cent seize médecins $(37 \%)$ avaient proposé un opioïde associé ou non à un 
Tableau 1 Distribution des principaux antalgiques et sédatifs administrés lors d'une analgésie-sédation procédurale

\begin{tabular}{|ll|}
\hline Traitement $(\boldsymbol{n}=\mathbf{3 0 8})$ & Valeurs \\
\hline Opioïdes & \\
Morphine & $232(75)$ \\
Sufentanil & $26(8)$ \\
Fentanyl & $14(5)$ \\
Sédatifs & \\
Kétamine & $163(53)$ \\
Midazolam & $109(35)$ \\
Propofol & $15(5)$ \\
MEOPA & $30(10)$ \\
Bloc iliofascial & $131(42)$ \\
\hline MEOPA : mélange équimolaire d'oxygène et de protoxyde \\
d'azote \\
Les données sont exprimées en nombre (pourcentage)
\end{tabular}

antalgique de palier 1, à du MEOPA ou à une ALR, et 47 médecins (15\%) avaient proposé un sédatif associé ou non à un antalgique de palier 1 , à du MEOPA ou à une ALR.

Le médecin n'ayant utilisé ni opioïde ni sédatif avait réalisé une ALR associée à du paracétamol.

La morphine avait été titrée par 229 médecins (99\%). Les posologies proposées en dose initiale étaient de $0,1 \mathrm{mg} / \mathrm{kg}$, $2 \mathrm{mg}$ ou $3 \mathrm{mg}$ dans $93 \%$ des cas. Après la dose initiale, $68 \%$ des médecins administraient des bolus de titration de 2 ou $3 \mathrm{mg}$. La kétamine avait été utilisée en titration par 25 sur 163 médecins (15\%), avec des doses très variables. Cinquante-trois médecins (32\%) avaient utilisé des posologies de kétamine supérieures ou égales à $0,5 \mathrm{mg} / \mathrm{kg}$ et 75 (46\%), des doses inférieures (dose inconnue pour trois médecins). Le propofol avait été administré en titration par 6 sur 15 médecins (40\%), 4 (26\%) l'utilisaient sans connaître la posologie et $6(40 \%)$ à une dose comprise entre 0,5 et $1 \mathrm{mg} / \mathrm{kg}$.

Quatre-vingt-trois médecins (27 \%) connaissaient les recommandations et les avaient bien citées dans un second temps. Quatre-vingt-cinq (27 \%) Smur possédaient un protocole d'analgésie-sédation.

Tous les médecins avaient dans leurs UMH du paracétamol, de la morphine, du midazolam et de la kétamine. Aucun médecin n'a déclaré avoir du propofol. Cent quatrevingt-neuf médecins (61\%) pratiquaient l'ALR en extrahospitalier, dont le bloc iliofascial (BIF) pour tous et des blocs du poignet et axillaires pour 4 d'entre eux $(2 \%)$. Neuf $(5 \%)$ Smur possédaient un échographe pour les ALR et huit ( $4 \%$ ) avaient un neurostimulateur. L'anesthésique local le plus utilisé était la lidocaïne (144 [76\%]). Cent quarante-sept (48 \%) médecins déclaraient avoir du MEOPA dans leur Smur, parmi lesquels 132 (90\%) l'utilisaient principalement en traumatologie, 64 (43\%) en pédiatrie et $24(16 \%)$ lors de la réalisation de soins.

Pour l'évaluation de la douleur des patients communicants, 163 médecins (53\%) utilisaient l'échelle numérique (EN), 125 (40\%) l'échelle visuelle analogique (EVA) et 20 (6 \%) l'échelle verbale simple. Un médecin n'utilisait aucune échelle. Pour les adultes non communicants, 198 (64\%) des médecins n'utilisaient aucune échelle. Pour les enfants, 159 médecins (51\%) utilisaient l'échelle des visages, et 94 (30\%) n'utilisaient aucune échelle. Cent vingtneuf médecins (42\%) avaient utilisé en plus de l'autoévaluation, une hétéroévaluation pour évaluer l'efficacité de l'analgésie de leur patient. Pour 113 médecins (39\%), la douleur était contrôlée quand l'EVA ou l'EN était inférieure à 3. Quarante-quatre médecins $(15 \%)$ avaient utilisé une échelle pour évaluer la profondeur de la sédation, dont 41 le score de Ramsay (13\%), 3 (1\%) le score de Glasgow et 1 , une échelle créée localement par les urgentistes et les médecins anesthésistes.

\section{Discussion}

Les résultats de cette enquête nous montrent qu'il y a une grande hétérogénéité des pratiques médicales lorsqu'il s'agit de réaliser une ASP pour l'exécution d'une procédure douloureuse en extrahospitalier.

La limite principale de l'étude repose sur le fait que pour chaque centre un seul médecin était interrogé, celui-ci ne représentant que lui-même et non pas tous ses collègues du centre en question. Le panel finalement obtenu n'est peutêtre pas tout à fait représentatif de l'ensemble des urgentistes travaillant dans un Smur. La seconde limite est liée au fait qu'il ne s'agit que de réponses déclarées et non pas le constat d'une situation réelle. Il est impossible de dire si les modalités de prise en charge proposées par les médecins pour ce cas clinique théorique seraient les mêmes en situation clinique réelle. Cependant, les réponses obtenues étaient exhaustives. Ces résultats viennent renforcer d'une autre manière ceux obtenus dans un travail évaluant les modalités ASP dans 10 Smur français [4]. Lors de 210 interventions, 25 combinaisons différentes associant antalgiques et/ou sédatifs avaient été utilisées [4]. Ce manque d'homogénéité se retrouve dans notre étude et pourrait être rattaché d'une part à un défaut de connaissance des recommandations et d'autre part à l'absence de protocole local.

La prescription de morphine et son mode d'administration (doses et titration) étaient majoritairement en accord avec les RFE de 2010 [2]. Pour la réalisation des procédures désagréables, un sédatif était administré dans deux tiers des cas en accord avec les recommandations. Dans le travail observationnel réalisé dans 10 Smur français, un sédatif était utilisé dans $55 \%$ des cas [4]. Lors de la réalisation de la 
procédure, le taux de douleurs intenses à sévères était de $27 \%$ lors de l'utilisation d'un sédatif contre $68 \%$ chez ceux n'en recevant pas [4]. L'utilisation d'un sédatif et la gestion efficace de la douleur dans cette situation n'ont pas qu'un impact immédiat. Il a été en effet montré que le souvenir de la procédure était significativement associé à la profondeur de la sédation, l'intensité de la douleur et à la satisfaction du patient [5]. Dans notre enquête, la kétamine était le sédatif le plus utilisé, mais à des posologies inférieures à celles proposées par les RFE $(0,5$ à $1 \mathrm{mg} / \mathrm{kg})$ dans deux tiers des cas. Cependant, la kétamine était aussi proposée dans les RFE à des doses plus faibles $(0,1$ à $0,3 \mathrm{mg} / \mathrm{kg})$ dans le cadre de la prise en charge de la douleur spontanée [2]. Cela est peut-être à la source d'une confusion concernant les posologies. Le propofol était par contre très peu utilisé et avec les posologies recommandées dans seulement $40 \%$ des cas. Une étude qualitative réalisée en 2016 dans des services d'urgences pédiatriques de Grande-Bretagne et d'Irlande montrait que l'utilisation de sédatifs à des posologies insuffisantes était liée à une crainte des effets indésirables et à l'absence d'apprentissage de ces procédures lors des études universitaires [6]. Il a été pourtant montré que la kétamine et le propofol pouvaient être utilisés en toute sécurité dans la mesure où les procédures et les modalités étaient respectées. En 2008, un travail avait montré que l'utilisation de la kétamine pour l'analgésie-sédation en extrahospitalier n'était pas associée à une augmentation significative des complications [7]. Cela a été confirmé par une revue récente de la littérature faisant le point sur les complications associées à l'analgésiesédation en urgence avec la kétamine et le propofol [8].

Peu de Smur possédaient un protocole d'analgésiesédation. Déjà en 2004, seulement $37 \%$ des Smur avaient un protocole de prise en charge de la douleur [9]. Plusieurs travaux ont pourtant bien montré que la mise en place d'un protocole était associée à une amélioration de la prise en charge de la douleur. En extrahospitalier, les patients traités selon un protocole d'analgésie par morphine en intraveineux étaient mieux et plus rapidement soulagés que ceux traités par les médecins n'utilisant pas de protocole [10]. Un travail plus ancien avait déjà montré l'amélioration de la prise en charge de la douleur en extrahospitalier après mise en place d'une formation de deux semaines et d'un protocole d'analgésie [11]. L'ALR prend de plus en plus de place en médecine d'urgence et en médecine préhospitalière pour la prise en charge de la douleur. Le taux de succès du BIF réalisé par les smuristes pour l'analgésie d'une fracture fémorale est de $94 \%$ [12]. Cependant, moins de la moitié des médecins de notre enquête l'avaient utilisé malgré l'indication évidente. Il a été récemment montré que la moitié des urgentistes manquait de formation sur les pratiques de l'ALR [13]. Le MEOPA n'était présent que dans la moitié des Smur malgré son efficacité reconnue dans certaines indications et malgré les RFE $[2,14]$. Notre travail a montré que l'évaluation de la douleur des patients communicants était réalisée en accord avec les RFE [2]. Par contre, pour les patients non communicants, aucune échelle n'était proposée dans deux tiers des cas. Deux échelles comportementales ont été validées pour l'évaluation de la douleur aiguë, Algoplus pour les patients non communicants de plus de 65 ans et BOS-3 pour tous les adultes non communicants $[15,16]$. Pour les enfants non communicants en situation d'urgence, l'échelle validée est EVENDOL [17,18]. L'hétérogénéité des procédures antalgiques proposées peut en partie être expliquée par le fait que deux tiers des médecins ne connaissaient pas les RFE. En 2004, la moitié ne connaissait pas les recommandations de l'époque sur le même sujet [10].

\section{Conclusion}

Cette étude a mis en évidence une grande hétérogénéité des modalités de réalisation de l'ASP en extrahospitalier, avec notamment des difficultés de maniement des sédatifs. Par ailleurs, les modalités d'évaluation de la douleur des patients les plus vulnérables (non communicants) n'étaient pas connues. L'absence de protocole dans de nombreux Smur et la méconnaissance des recommandations peuvent en partie expliquer ces résultats.

Liens d'intérêts : les auteurs déclarent ne pas avoir de lien d'intérêt.

\section{Références}

1. Godwin SA, Burton JH, Gerardo CJ, et al (2014) Clinical policy: procedural sedation and analgesia in the emergency department. Ann Emerg Med 63:247-58

2. Vivien B, Adnet F, Bounes V, et al (2012). Sédation et analgésie en structure d'urgence. Réactualisation de la conférence d'experts de la SFAR de 1999. Ann Fr Anesth Reanim 31:391-404

3. Société française d'anesthésie et de réanimation, Samu de France, Société francophone de médecine d'urgence (2004) Pratique des anesthésies locales et locorégionales par des médecins non spécialisés en anesthésie-réanimation, dans le cadre des urgences. Ann Fr Anesth Reanim 23:167-76

4. Galinski M, Hoffman L, Bregeaud D, et al (2018). Procedural sedation and analgesia in trauma patients in an out-of-hospital emergency setting. A prospective multicenter observational study. Prehosp Emerg Care 22:497-505

5. Freeston JA, Leal A, Gray A (2012) Procedural sedation and recall in the emergency department: the relationship between depth of sedation and patient recall and satisfaction (a pilot study). Emerg Med J 29:670-2

6. McCoy S, Lyttle MD, Hartshorn S, et al (2016) A qualitative study of the barriers to procedural sedation practices in paediatric emergency medicine in the UK and Ireland. Emerg Med J 33:527-32

7. Bredmose PP, Lockey DJ, Grier G, et al (2009). Pre-hospital use of ketamine for analgesia and procedural sedation. Emerg Med J 26:62-4 
8. Bellolio MF, Gilani WI, Barrionuevo P, et al (2016) Incidence of adverse events in adults undergoing procedural sedation in the emergency department: a systematic review and meta-analysis. Acad Emerg Med 23:119-34

9. Galinski M, Ruscev M, Pommerie F, et al (2004) Prise en charge de la douleur aiguë sévère chez l'adulte en médecine préhospitalière : enquête nationale auprès des médecins de Smur. Ann Fr Anesth Reanim 23:1149-54

10. Ricard-Hibon A, Belpomme V, Chollet C, et al (2008) Compliance with a morphine protocol and effect on pain relief in out-of-hospital patients. J Emerg Med 34:305-10

11. Ricard-Hibon A, Chollet C, Saada S, et al (1999) A quality control program for acute pain management in out-of-hospital critical care medicine. Ann Emerg Med 34:738-44

12. Gozlan C, Minville V, Asehnoune K, et al (2005) Bloc iliofascial en médecine préhospitalière pour les fractures du fémur. Ann Fr Anesth Reanim 24:617-20

13. Violeau M, Lefort H, Mimoz O, et al (2015) Évaluation de la pratique des techniques d'anesthésie locorégionale en dehors de l'hôpital et à l'hôpital par les urgentistes d'une région française. Anesth Reanim 1:232-7

14. Ducassé JL, Siksik G, Durand-Béchu M, et al (2013) Nitrous oxide for early analgesia in the emergency setting: a randomized, doubleblind multicenter prehospital trial. Acad Emerg Med 20:178-84

15. Rat P, Jouve E, Pickering G, et al (2011) Validation of an acute pain-behavior scale for older persons with inability to communicate verbally: algoplus. Eur J Pain 15:198.e1-e10

16. Frasca M, Burucoa B, Domecq S, et al (2017) Validation of the behavioural observation scale 3 for the evaluation of pain in adults. Eur J Pain 21:1475-84

17. Fournier-Charrière E, Tourniaire B, Carbajal R, et al (2012) EVENDOL, a new behavioral pain scale for children of 0 to 7 in the emergency department: design and validation. Pain 153:1573-82

18. Beltramini A, Galinski M, Chabernaud JL, et al (2016) Pain assessment in children younger than 8 years in out-of-hospital emergency medicine: reliability and validity of EVENDOL score. Pediatr Emerg Care [Epub ahead of print] 